\title{
Arterial versus Venous Fluid Resuscitation; Restoring Cardiac Contractions in Cardiac Arrest Following Exsanguinations
}

\author{
Asser M. Youssef,," Alireza Hamidian Jahromi, ${ }^{2}$ and Cuthbert O. Simpkins ${ }^{3}$ \\ ${ }^{1}$ Chandler Regional Medical Center, College of Medicine-Phoenix, University of Arizona, Arizona, USA \\ ${ }^{2}$ Department of Surgery, Louisiana State University Health Sciences Center-Shreveport, Los Angeles, USA \\ ${ }^{3}$ Vivacelle Bio, Inc. Enterprise Works Chicago, Chicago, Illinois, USA \\ "Corresponding author: Asser M. Youssef, Chandler Regional Medical Center, College of Medicine-Phoenix, University of Arizona, Arizona, USA. Tel: +1-9174061309, \\ +1-3186756355, Fax: +1-3186754689, E-mail: asseryou@gmail.com
}

Received 2015 May 31; Revised 2015 August 21; Accepted 2015 November 20.

\begin{abstract}
Background: Arterial cannulation and intra-arterial (IA) fluid and blood resuscitation in the patients with severe shock is an easier approach compared with the intravenous (IV) access if concerns regarding the efficiency and safety of this approach are addressed. Objectives: We hypothesized that IA fluid resuscitation is more effective than IV resuscitation in restoring cardiac contractions (CC) of cardiac-arrested mice following severe hemorrhagic shock.

Methods: Mice $(\mathrm{N}=22)$ were anesthetized using ketamine/xylazine. Arterial and venous systems accessed through cannulation of the carotid artery and the Jugular vein, respectively. As much blood as possible was aspirated from the carotid artery access. Mice were observed until the complete cessation of chest wall motions. Following 30 seconds delay, IV $(\mathrm{N}=5)$ and IA access $(\mathrm{N}=6)$ were used for fluid resuscitation using Ringer Lactate (RL) in a similar volume to the aspirated blood. Mice were observed for restoration of chest wall motions. In phase-IIof the study, after cessation of chest motions, mice $(\mathrm{N}=11)$ underwent a thoracotomy and CCs were observed. In three mice, IV RL Infusion after cardiac arrest failed to restore CCs and was followed by IA RL infusion. In eight mice, following cardiac arrest intermittent IA RL infusion was performed.

Results: While IV RL Infusion failed to restore chest motion in mice $(\mathrm{N}=5)$, IA RL infusion restored chest motion in all mice examined $(\mathrm{N}=6)(\mathrm{P}=0.0067)$. In three mice, IV RL infusion after cardiac arrest showed no effect on CC. After failure of venous infusion, IA RL infusion was performed which resulted in restoration of CC for $13.33 \pm 1.76$ minutes. In eight mice, intermittent IA infusion of RL after cardiac arrest, sustained CC for $31.43 \pm 10.9$ minutes $(\mathrm{P}=0.017)$.

Conclusions: IA fluid resuscitation is superior to IV resuscitation in hemorrhagic shock induced cardiac arrest.
\end{abstract}

Keywords: Arterial, Venous, Fluid Resuscitation, Cardiac Contractions, Cardiac Arrest, Exsanguinations

\section{Background}

Hemorrhagic shock is a common form of hypovolemic shock, the most common type of shock, and in extreme situations can lead to a cardiac arrest. The rescue strategy in these cases includes stopping the bleeding source and blood and fluid resuscitation along with a complete cardiopulmonary resuscitation to return cardiac activity (electrical and mechanical). It is a universal practice to use large peripheral or central veins as an access to deliver blood, fluids and other resuscitation medications in these situations. In the extremely severe cases of hemorrhagic shock (Class IV), severe peripheral vascular constriction secondary to the compensatory neuronal and humoral mechanisms is present, which can potentially interfere with the process of intravenous (IV) cannulation. Arterial cannulation and intra-arterial (IA) fluid and blood resuscitation in the patients with severe shock is arguably an easier approach if concerns regarding the efficiency and safety of this approach are addressed. IA administration of the fluid might not only be easier to implement, but may be more effective as well.

The initial experiments comparing IA versus IV transfusion in cardiac arrest from exsanguination goes back more than half a century to when Case et al. (1953); Negovsky et al. (1962) and Kirmli et al. (1965) each reported their resuscitation of animals in cardiac arrest models $(1,2)$. Although the results were, to some extent, promising and showed a significantly superior outcome in the IA resuscitation group compared to their IV counterparts, the clinical practice never shifted in that direction. All the previous experiments in resuscitation used epinephrine in combination with the blood or fluid, but the question as to whether IA fluid resuscitation alone is effective enough in restoring cardiac contractions (CCs) in cardiac arrested victims following exsanguinations and is superior to IV fluid resuscitation remains unanswered. 


\section{Objectives}

In the current study, we hypothesized that IA fluid resuscitation is more effective than an IV fluid resuscitation in restoring cardiac contractions (CC) of cardiac-arrested mice following hemorrhagic shock.

\section{Methods}

All animal procedures were approved by the Louisiana State University Animal Use Committee. Male CD-1 mice from Charles River (Wilmingtom, Mass) weighting 27 47grams were used. Mice $(\mathrm{N}=22)$ were anesthetized using ketamine/xylazine administered subcutaneously.

\subsection{Vascular Access in Mice}

Once anesthetized, a longitudinal incision was made in the neck and the jugular vein and the carotid artery were defined. Arterial and venous systems were accessed through cannulation (under direct vision and using a microscope) of the carotid artery and the Jugular vein, respectively.

\subsection{Respiratory Arrest Model}

Blood was aspirated from the carotid artery access over one minute period. Mice $(\mathrm{N}=11)$ were then observed until complete cessation of the chest wall motions (Figure 1).

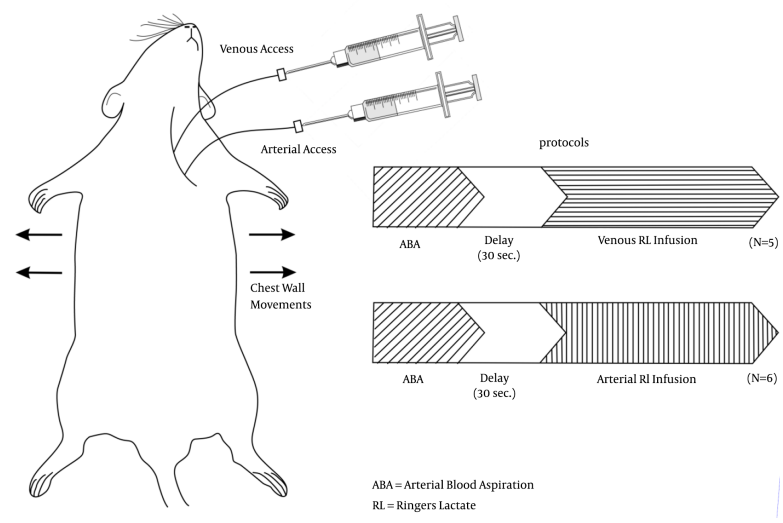

Figure 1. Schematic View Showing the Protocols Used in the Part One of the Study

\subsection{Fluid Resuscitation Protocol (Study Phase-I)}

RL was used for resuscitation in all mice. The amount infused was equal to the amount of blood withdrawn from the same mouse. Fluid resuscitation with RL began 30 seconds after complete cessation of respiratory movements.
IV access $(\mathrm{N}=5)$ and IA access $(\mathrm{N}=6)$ were used for fluid resuscitation using Ringer Lactate (RL) solutions in a similar volume to the aspirated blood (using a syringe). Mice were observed for any restoration of chest wall motions.

\subsection{Cardiac Arrest Model}

Blood was aspirated from the carotid artery access over one minute period. Mice $(\mathrm{N}=11)$ had midline thoracotomy and cardiac contractions were directly observed (Figure 2). Mice were then observed until cessation of the cardiac contractions (complete cessation of ventricular contractions)

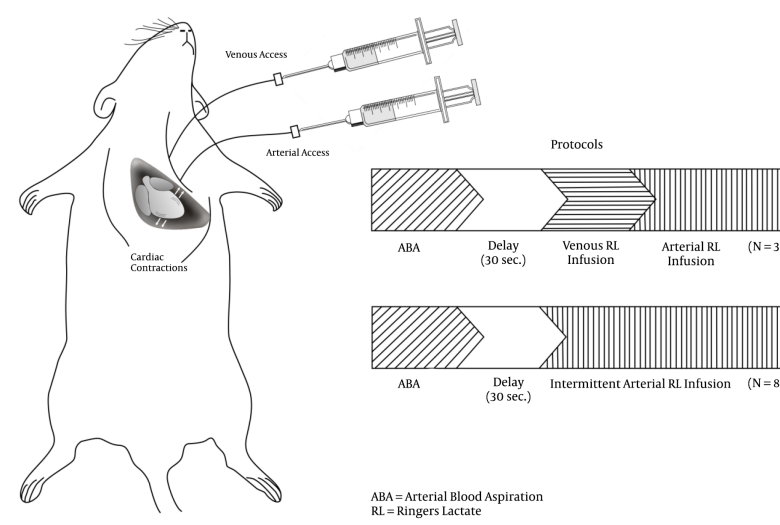

Figure 2. Schematic View Showing the Protocols Used in the Part Two of the Study

\subsection{Fluid Resuscitation Protocol (Study Phase-II)}

RL was used for resuscitation in all mice. The amount infused was equal to the amount of blood withdrawn from the same mouse (using a syringe). Fluid resuscitation with RL began 30 seconds after complete cessation of cardiac contractions. In three mice, IV infusion of RL after cardiac arrest failed to restore CCs and was followed by RL infusion via the carotid artery. In eight mice, intermittent IA infusion of RL after cardiac arrest was performed.

\section{Results}

\subsection{Resuscitation After Respiratory Arrest (Study Phase-I)}

As much as possible blood was aspirated from the carotid artery. Chest wall movements were observed. After cessation of chest wall movement, RL was infused IV ( N $=5)$ and IA $(N=6)$. While Intravenous RL infusion failed to restore chest wall motion in mice $(\mathrm{N}=5)$, IA infusion of $\mathrm{RL}$ restored chest wall motion in all of the mice $(\mathrm{N}=6)$ examined $(\mathrm{P}=0.0067)$. 


\subsection{Resuscitation After Cardiac Arrest (Study Phase-II)}

In three mice, 30 seconds after cardiac arrest, RL was infused through IV access in an amount equal to the volume of withdrawn blood. There were no restoration of CCs. Following that RL was infused through IA access which caused restoration of $C C$ for 14,10 and 16 minutes in mice \# 1,2 and 3 respectively (mean $+\mid$ - SE of $13.33+/-1.76$ minutes). In eight other mice intermittent IA infusion of RL after cardiac arrest, sustained CC for a mean + - SE of $31.43+\mid-10.9$ minutes $(\mathrm{P}=0.017)$.

\section{Discussion}

Currently, the intra-arterial route is only utilized for drug administration in specific clinical situations like vasodilator administration in the treatment of vasospasm and thrombolytic drugs for embolic events $(3,4)$. Specific characteristics of IA access, including the higher risk of bleeding from the puncture site, potential limb ischemia secondary to artery spasm and the possibility of embolic events, as well as lack of particle filtration effects of lung and liver in this method, are some of the factors that make this access less favorable for routine use. The mentioned associated risks would be justifiable if the success rate of a life saving procedure, fluid resuscitation in a severe hypovolemic shock victim with cardiac arrest, improves significantly. Eisenburger et al. (2000) (5) reported a case where inadvertent placement of a central line and IA administration (aortic arch) of excessive amount of epinephrine during cardiopulmonary resuscitation led to rhabdomyolysis and acute renal failure. The patient recovered and the renal failure was resolved after a period of hemodialysis. The authors attributed this complication to the IA route of epinephrine administration, but one could argue that the mentioned complication could well be attributed to the high dose of epinephrine rather than the administration route. Even if we accept that the IA route of resuscitation could potentially have such complications; eminent superior success of a life saving procedure, resuscitation through this route outweighs the potential risks. Our data supports the findings of the previously reported experiments $(1,2,6,7)$ that IA fluid infusion is superior to IV infusion in resuscitation efforts for cardiac arrest induced by hemorrhagic shock. The rapid rise in the intra-aortic pressure following IA infusion (hydraulic effect) and immediate direct coronary blood flow as opposed to IV pooling in the distensible venous bed (IV infusion) is the proposed mechanism to explain these findings (8). Contrary to the previous experiments, our study assessed the impact of IA fluid (RL) resuscitation alone, with no additional epinephrine, in restoring CCs in cardiac arrested victims following exsanguinations. This route of administration was proved to be effective and helpful.

We used intermittent fluid resuscitation as in reality using a single crystalloid bolus may not be adequate to maintain intra-vascular volume in the absence of blood transfusion. A significant portion of transfused fluid will move to the interstitial space. This type of fluid resuscitation replicates the situation in the real life resuscitations.

In our experiment, IA infusion, even following a failed IV resuscitation attempt, gave the mice $(\mathrm{N}=3)$ a chance to regain their CCs for a period of time; however, the CCs in this group continued for a significantly shorter period of time compared with those mice who received intermittent IA resuscitation $(\mathrm{N}=8)$. This finding signifies the importance of an early restoration of fluid resuscitation using an IA access route rather the delayed IA usage in case of IV resuscitation failure.

Further studies using a larger number of cases are required to assess this hypothesis further. This subject needs to be revisited by the medical community, and there might be reasonable room for the resuscitation guidelines and clinical practice shifting towards using IA administration routinely in cardiopulmonary resuscitation.

\subsection{Conclusion}

IA infusion is superior to IV resuscitation in hemorrhagic shock induced cardiac arrest. Early restoration of fluid resuscitation using an IA route is of utmost importance in increasing the chance of recovery in a case with cardiac arrest secondary to exsanguinations.

\section{Acknowledgments}

The authors would like to thank Mr. John Cyrus and Ms. Talicia Tarver who provided us editorial assistance.

\section{Footnote}

Authors' Contribution: All the authors have contributed towards performing the research and preparation of the manuscript

\section{References}

1. Case RB, Sarnoff SJ, Waithe PE, Sarnoff LC. Intra-arterial and intravenous blood infusion in hemorrhagic shock; comparison of effects on coronary blood flow and arterial pressure. J Am Med Assoc. 1953;152(3):208-12. [PubMed: 13044499].

2. Kirmli B, Safar P. Arterial versus venous transfusion in cardiac arrest from exsanguination. Anesth Analg. 1965;44(6):819-30. [PubMed: 5891910]. 
3. French KF, Hoesch RE, Allred J, Wilder M, Smith AG, Digre KB, et al. Repetitive use of intra-arterial verapamil in the treatment of reversible cerebral vasoconstriction syndrome. J Clin Neurosci. 2012;19(1):174-6. doi: 10.1016/j.jocn.2011.06.016. [PubMed: 22118796].

4. Kuhn JP, Hoene A, Miertsch M, Traeger T, Langner S, Hosten N, et al. Intraarterial recombinant tissue plasminogen activator thrombolysis of acute and semiacute lower limb arterial occlusion: quality assurance, complication management, and 12-month follow-up reinterventions. AJR Am J Roentgenol. 2011;196(5):1189-93. doi: 10.2214/AJR.10.4477. [PubMed: 21512091].

5. Eisenburger P, Laggner AN, Lenz K, Druml W. Acute renal failure and rhabdomyolysis after inadvertent intra-arterial infusion of excessive doses of epinephrine during cardiopulmonary resuscitation. Wien Klin Wochenschr. 2000;112(4):174-6. [PubMed: 10726331].

6. Negovsky VA, Shikunova LG, Gurvich NL, Riabova NM. Arterial versus venous transfusion in cardiac arrest from exsanguination: further studies in dogs. Anesth Analg. 1972;51(2):251-7. [PubMed: 5062128].

7. Kirimli B, Kampschulte S, Safar P. Cardiac arrest from exsanguination in dogs. Evaluation of resuscitation methods. Acta Anaesthesiol Scand Suppl. 1968;29:183-9. [PubMed: 4877220].

8. Gentile NT, Martin GB, Appleton TJ, Moeggenberg J, Paradis NA, Nowak RM. Effects of arterial and venous volume infusion on coronary perfusion pressures during canine CPR. Resuscitation. 1991;22(1):55-63. [PubMed: 1658894]. 